\title{
INFLUÊNCIA DAS VARIÁVEIS DE PROCESSO NA FORMAÇÃO DE MACRO DEFEITOS DURANTE PROCESSAMENTO POR FRICÇÃO E MISTURA*
}

\author{
Angelo Siqueira da Silva ${ }^{1}$ \\ Saulo Brinco Diniz ${ }^{2}$ \\ Andrey de Moraes Barcelos Casanova ${ }^{3}$ \\ Andersan dos Santos Paula ${ }^{4}$ \\ Luiz Paulo Mendonça Brandão ${ }^{5}$
}

\section{Resumo}

O processo de fricção e mistura, tem sido largamente estudado devido as microestruturas resultantes após o processamento, no qual são fortemente influenciadas pelos parâmetros utilizados durante o processamento. Este trabalho teve por objetivo avaliar a influência das variáveis de processo: rotações por minutos, velocidade de avanço, inclinação e penetração da ferramenta no material, na formação de macro defeitos em uma liga de alumínio 7475-T7351 em decorrência do processamento por fricção e mistura. A ação de caracterização e localização do defeito deu-se por meio de vídeo estereoscópio, onde foi possível observar a maior incidência do defeito de volume na zona de mistura (Cavidade), assim como uma preferência assimétrica quanto à localização do macro defeito no lado de retrocesso da ferramenta.

Palavras-chave: Processamento por Fricção e Mistura; Macro Defeitos; Zona de Mistura.

\section{INFLUENCE OF PROCESS VARIABLES ON MACRO DEFECTS FORMATION DURING THE FRICTION STIR PROCESSING}

\section{Abstract}

The friction stir processing has been largely studied due to the microstructures resulting after processing, whose are strongly influenced by the parameters used during the processing. This study aimed to evaluate the influence of the process parameters: rotation per minutes, feed speed, tool tilt and penetration, in the formation of macro defect in a 7475-T7351 aluminum alloy due to friction stir processing. An action of characterization and defect location occurred by means of video stereoscope, where were possible detect a greater incidence of volume defect in the stir zone (Cavity), as well as an asymmetric preference as to the location of the macro defect in the recoil side of the tool.

Keywords: Friction Stir Processing; Macro Defects; Nugget.

1 Eng. Metalurgista, Mestrando em Ciência dos Materiais (PGCM/SE-4), IME, Rio de Janeiro - RJ.

2 M.Sc., Eng. Metalurgista, Doutorando em Ciência dos Materiais (PGCM/SE-4), IME, Rio de Janeiro - RJ, Brasil; Professor DI - 1, CEFET - Angra dos Reis, Angra dos Reis, RJ, Brasil.

3 M.Sc., Tecnólogo em Processos Metalúrgicos, Doutorando em Ciência dos Materiais (PGCM/SE4), IME, Rio de Janeiro - RJ, Brasil.

4 D.Sc.. M.Sc., Eng. Metalurgista, Professora Adjunta (PGCM/SE-4), IME, Rio de Janeiro - RJ e orientadora credenciada do PPGEM/UFF, Volta Redonda, RJ, Brasil.

5 D.Sc., M.Sc., Eng. Metalurgista, Professor Associado (PGCM/SE-4), IME, Rio de Janeiro - RJ. 


\section{INTRODUÇÃO}

O conceito básico do processo de união por friç̧ão (Friction Stir Welding - FSW) e/ou processo de fricção e mistura (Friction Stir Processing - FSP), consiste no avanço e na rotação em alta velocidade de uma ferramenta constituída de um ombro e um pino não consumível especialmente desenvolvido para tal atividade. A referida ferramenta possui duas funções essenciais: (a) aquecimento do material de trabalho, e (b) movimento de material para produzir a junta. Durante o FSW, o aquecimento localizado é realizado pela fricção entre a ferramenta e o material a ser processado, e com isso ocorre a elevação da temperatura em torno 0,8Tf (temperatura de fusão), levando ao coalescendo local deste material após o resfriamento, conforme trabalho de LI et al. [1]. Assim durante este processo de soldagem, o material sofre uma deformação plástica acentuada devido à ação do fenômeno de coalescimento e rotação da ferramenta, resultando na geração de grãos recristalizados finos e equiaxiais. A soldagem por friç̧ão produz excelentes propriedades mecânicas no cordão de solda e com isso, o processo FSW é considerado o desenvolvimento mais significativo na união de metais, o qual foi inicialmente desenvolvido (em 1991 pelo The Welding Institute (TWI) do Reino Unido) como uma técnica de união no estado sólido com foco na baixa soldabilidade de ligas de alumínio via processo convencional de fusão.

Recentemente, o processamento por fricção e mistura (FSP) foi desenvolvido por R. S. Mishra [2] como uma ferramenta genérica para modificação microestrutural baseada nos princípios básicos da FSW. Neste caso, uma ferramenta rotativa é inserida numa peça de trabalho para modificação microestrutural localizada para a obtenção ou aprimoramento de propriedades específicas. Como por exemplo, a superplasticidade, ao qual foi obtida na liga comercial de alumínio 7075 por FSP [35]. Além disso, a técnica FSP tem sido usada para homogeneização de peças de alumínio produzidas via metalurgia do pó [6], modificação microestrutural de compósitos de matriz de metálica [7] e melhoria das propriedades mecânicas em ligas de alumínio fundido [8].

A microestrutura obtida através do processo por fricção e mistura possui as seguintes regiões conforme a figura 1 [12]:

Lado de retrocesso

(LR)

(B)

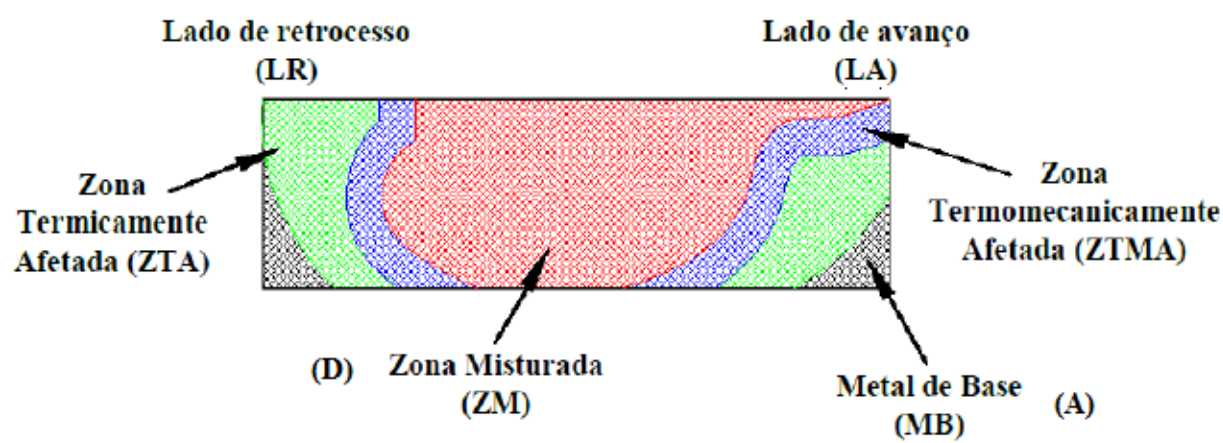

(MB)
(C)

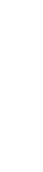

Figura 1. Aspecto macro das regiões com aspectos microestruturais distintos em uma seção transversal oriunda de um processo FSW [12].

- Região $A$ - Metal de Base (MB): região não afetada pelo calor ou deformação induzida pela ferramenta de soldagem;

- Região B - Zona Termicamente Afetada (ZTA): região afetada pelo ciclo térmico durante a soldagem, o que leva a modificação microestrutural e de propriedades mecânicas, porém sem deformação plástica residual na microestrutura; 
- Região C - Zona Termo-Mecanicamente Afetada (ZTMA): nesta área o material foi plasticamente deformado pela ferramenta e o fluxo de calor resultante exerceu algum tipo de influência no material que está próximo a área de atrito e de material extrudado.

- D - Zona Misturada (Weld Nugget): é a região central localizada dentro da ZTMA onde ocorre a recristalização, em que grãos originais e contornos de subgrãos favorecem a formação de finos grãos equiaxiais oriundos da recristalização.

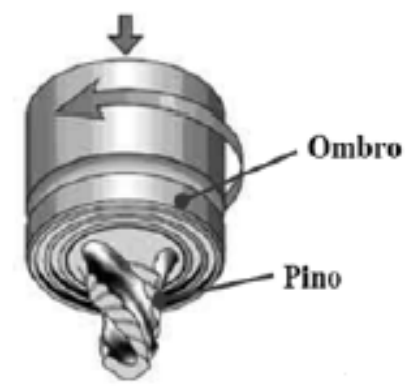

(a)

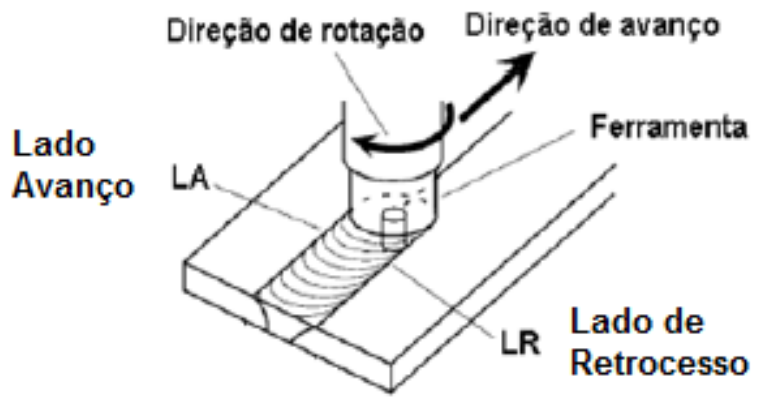

(b)

Figura 2. Figura esquemática (a) ferramenta e (b) do processo FSW e FSP [14].

As principais variáveis apresentadas para os processos FSW e FSP são: Projeto do pino da ferramenta (Pin); Velocidade de rotação da ferramenta (Tool rotation rate); Velocidade de deslocação da ferramenta (Tool traverse speed); Ângulo de inclinação da ferramenta (Tilt angle); Profundidade de imersão (Plunge depth).

Para o presente trabalho, a variável Projeto do Pino da ferramenta adotada não foi alterada. Logo, não houveram mudanças na geometria do pino e do ombro.

Para o processamento por fricção e mistura (FSP), tem-se como provável aparecimento de defeitos os seguintes: porosidade, trinca e defeitos de volume na zona de mistura a nível macroscópico. Estes defeitos induzem concentrações de tensões que podem ter uma influência negativa no comportamento mecânico do material, não atingindo assim o propósito do processamento. Com tudo, cabe salientar que a presença de descontinuidades decorrentes do processo por fricção, são inferiores quantitativamente na soldagem por atrito que nos processos típicos por fusão, segundo JAMES et al.[9].

As descontinuidades típicas que ocorrem no processo FSW e FSP resultam de projeto incorreto de ferramenta, da seleção de parâmetros inadequados, ou da combinação de problema de projeto de ferramenta com parâmetros de processo inadequados conforme descrito por LOMOLINO et al [10].

\section{MATERIAL E MÉTODOS}

O material utilizado neste estudo foi uma liga de alumínio 7475-T7351, primeiramente laminada a quente até $12,5 \mathrm{~mm}$, e posteriormente laminada a temperatura ambiente até $3,0 \mathrm{~mm}$ de espessura, com reduções por passe de aproximadamente $10 \%$.

Para o processamento por fricção e mistura (FSP) foi utilizado um equipamento de Friction Stir Processing de 5-eixos, com eixo-ferramenta de $30 \mathrm{~kW}$, força axial máxima de $80 \mathrm{kN}$ do Instituto de Pesquisas Tecnológicas - IPT - Núcleo de 
Estruturas Leves - LEL, instalado no Parque Tecnológico São José dos Campos (SP / Brasil), conforme fotografia apresentada na Figura 3.

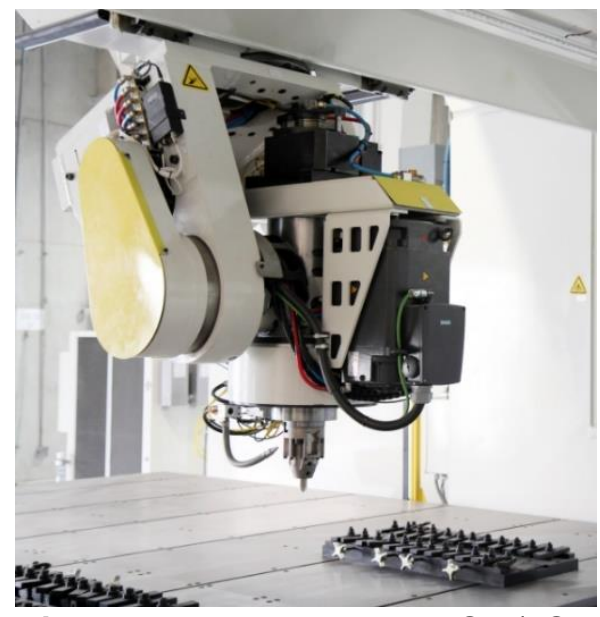

Figura 3. Equipamento de FSW/FSP

Com o objetivo de uma análise visual macro dos possíveis defeitos ou descontinuidades no material após processamento, seguiu-se a seguinte sequência de preparação das amostras: Lixamento com lixas de carbeto de silício de 240, 320, 400, 500, 600, 1000, 1200, 1500, 2000, 2500 mesh (Norma CAMI).

Para a análise visual macro da seção transversal do material utilizou-se o estereoscópio Carl Zeiss DV4 / Stemi 2000 / Axiover 40 para a aquisição de imagens com 8x de ampliação. Visando a simplificação da identificação das amostras, foi adotada para nomenclatura uma sigla composta pelos valores utilizados nas seguintes variáveis de processamento: rotações por minuto (RPM), velocidade de avanço na soldagem (VA), inclinação da ferramenta e profundidade aplicada pela ferramenta.

\section{XX/YYIZZWW/KK}

XX - Sigla da Amostra

YY - RPM
ZZ - Velocidade de Avanço ( $\mathrm{mm} / \mathrm{min})$

WW - Inclinação da Ferramenta $\left({ }^{\circ}\right)$

KK - Penetração da Ferramenta no Material

Adote-se com valor em centésimo de milímetro a penetração da ferramenta no material.

\section{RESULTADOS E DISCUSSÃO}

Para uma melhor análise quanto a influência das variáveis de processo, dividiu-se as amostras em 10 blocos, conforme tabela 1. A apresentação dos resultados quanto às inspeções visuais de superfície, raiz e presença de macro defeitos na amostra encontra-se descritos nas tabelas de 2 a 11. 
Tabela 1. Divisão das amostras em blocos de 1 a 10 para análise de resultados.

\begin{tabular}{cc}
\hline Blocos & \multicolumn{1}{c}{ Amostras } \\
\hline \multirow{2}{*}{1} & $\mathrm{~A} 1 / 350 / 50 / 3 / 0$ \\
\cline { 2 - 2 } & $\mathrm{A} 4 / 350 / 50 / 3 / 5$ \\
\cline { 2 - 2 } & $\mathrm{A} 5 / 350 / 50 / 3 / 10$ \\
\hline 2 & $\mathrm{~A} 4 / 350 / 50 / 3 / 5$ \\
\hline \multirow{2}{*}{3} & $\mathrm{~A} 2 / 350 / 100 / 3 / 5$ \\
\hline \multirow{2}{*}{4} & $\mathrm{~A} 3 / 500 / 100 / 3 / 5$ \\
\hline \multirow{2}{*}{5} & $\mathrm{~A} 7 / 500 / 50 / 3 / 5$ \\
\hline & $\mathrm{A} 6 / 500 / 50 / 0 / 0$ \\
\hline
\end{tabular}

\begin{tabular}{|c|c|}
\hline Blocos & Amostras \\
\hline \multirow{2}{*}{6} & $\mathrm{~A} 8 / 1000 / 50 / 0 / 0$ \\
\hline & A9/1000/50/0/5 \\
\hline \multirow{2}{*}{7} & A9/1000/50/0/5 \\
\hline & $\mathrm{A} 10 / 1000 / 100 / 0 / 5$ \\
\hline \multirow{2}{*}{8} & $\mathrm{~A} 10 / 1000 / 100 / 0 / 5$ \\
\hline & A11/1500/100/0/5 \\
\hline \multirow{2}{*}{9} & $\mathrm{~A} 11 / 1500 / 100 / 0 / 5$ \\
\hline & $\mathrm{A} 12 / 1500 / 100 / 0 / 8$ \\
\hline \multirow{3}{*}{10} & A2/350/100/3/5 \\
\hline & $\mathrm{A} 3 / 500 / 100 / 3 / 5$ \\
\hline & A11/1500/100/0/5 \\
\hline
\end{tabular}

As macrografias apresentadas na figura 4 representam as inspeções visuais de superfície e raiz das amostras 1,4 e 5 (Bloco 1 - Tabela 2), assim como a macroestrutura com ampliação de $8 x$ e com indicações dos lados de avanço (LA) e de retrocesso (LR).

Para as amostras do bloco 1, foi possível verificar que aumentos na profundidade de penetração da ferramenta no material, provocou uma tendência positiva na mistura do material na zona do nugget. A profundidade de penetração da ferramenta está associada à altura do pino, quando a profundidade de inserção é muito baixa, o ombro da ferramenta não entra em contato com a superfície original da peça. Assim, o ressalto rotativo não pode mover o material agitado eficientemente da frente para a parte de trás do pino, resultando na geração de descontinuidades, como por exemplo, o defeito de volume na zona de mistura [2]. Pode-se também observar que as descontinuidades concentraram-se de maneira assimétrica para o lado do retrocesso. Este fenômeno se faz presente à medida que a ferramenta se move para frente, através de uma zona de deformação inicial que se forma quando o material é aquecido abaixo da temperatura crítica ou quando não vence a tensão de fluxo crítico do material necessário para um fluxo de material eficiente [11].

Tabela 2. Parâmetros bloco 1 com variação da profundidade de penetração da ferramenta.

\begin{tabular}{lccccc} 
Nomenclatura & Amostra & RPM & $\begin{array}{c}\text { VA } \\
\text { (mm/min) }\end{array}$ & $\begin{array}{c}\text { Inclinação } \\
\text { (Graus) }\end{array}$ & $\begin{array}{c}\text { Profundidade de } \\
\text { Penetração da } \\
\text { ferramenta (mm) }\end{array}$ \\
\hline A1/350/50/3/0 & 1 & 350 & 50 & 3 & 0,00 \\
\hline $\mathrm{A} 4 / 350 / 50 / 3 / 5$ & 4 & 350 & 50 & 3 & 0,05 \\
\hline $\mathrm{A} 5 / 350 / 50 / 3 / 10$ & 5 & 350 & 50 & 3 & 0,10 \\
\hline
\end{tabular}




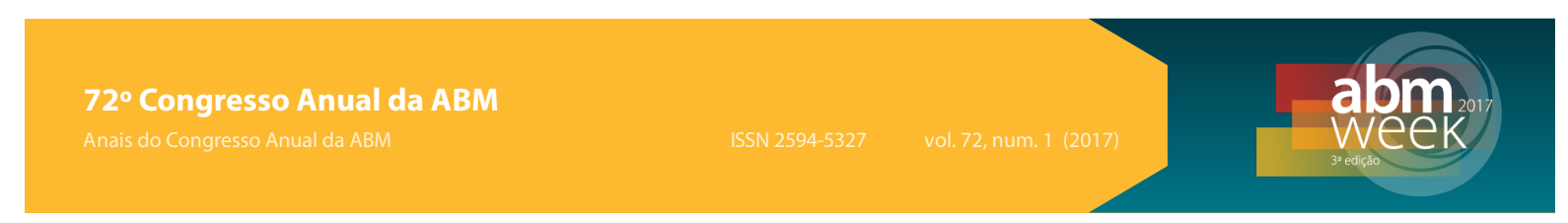

(a)
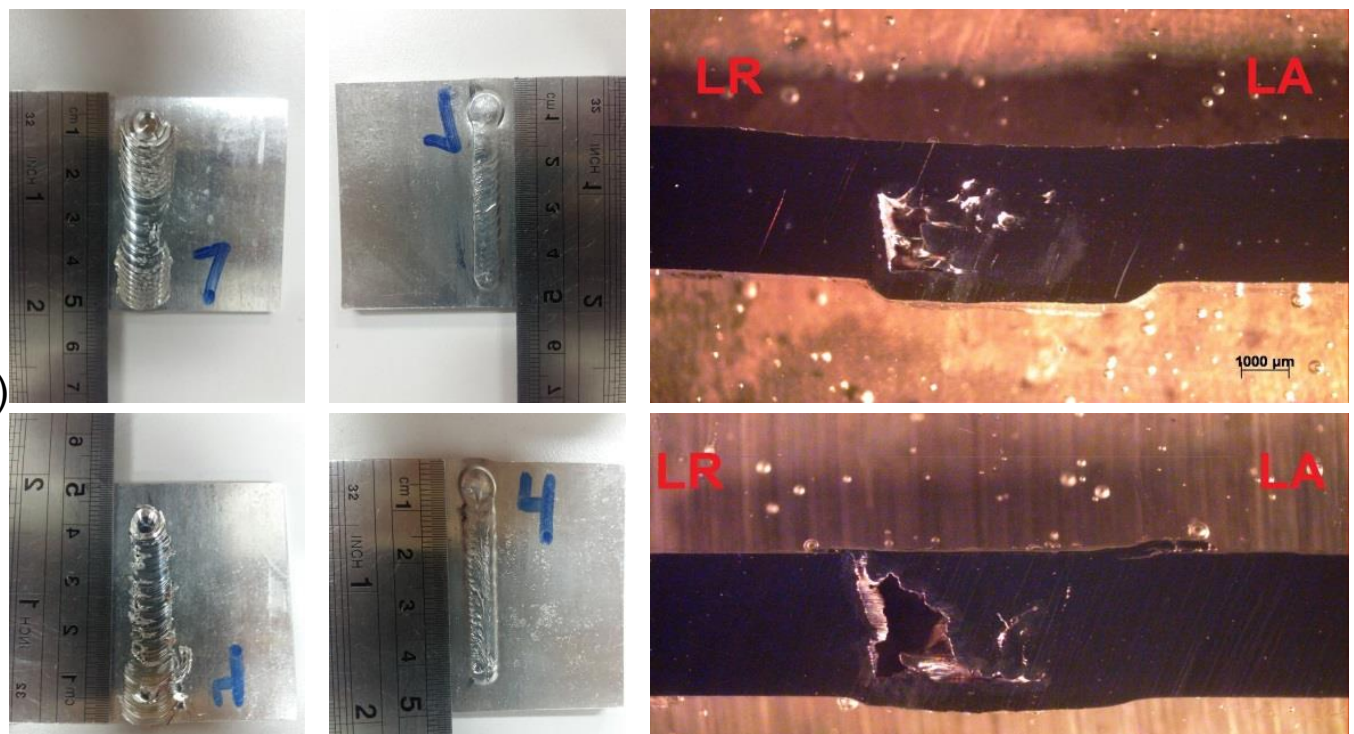

(b)

(c)
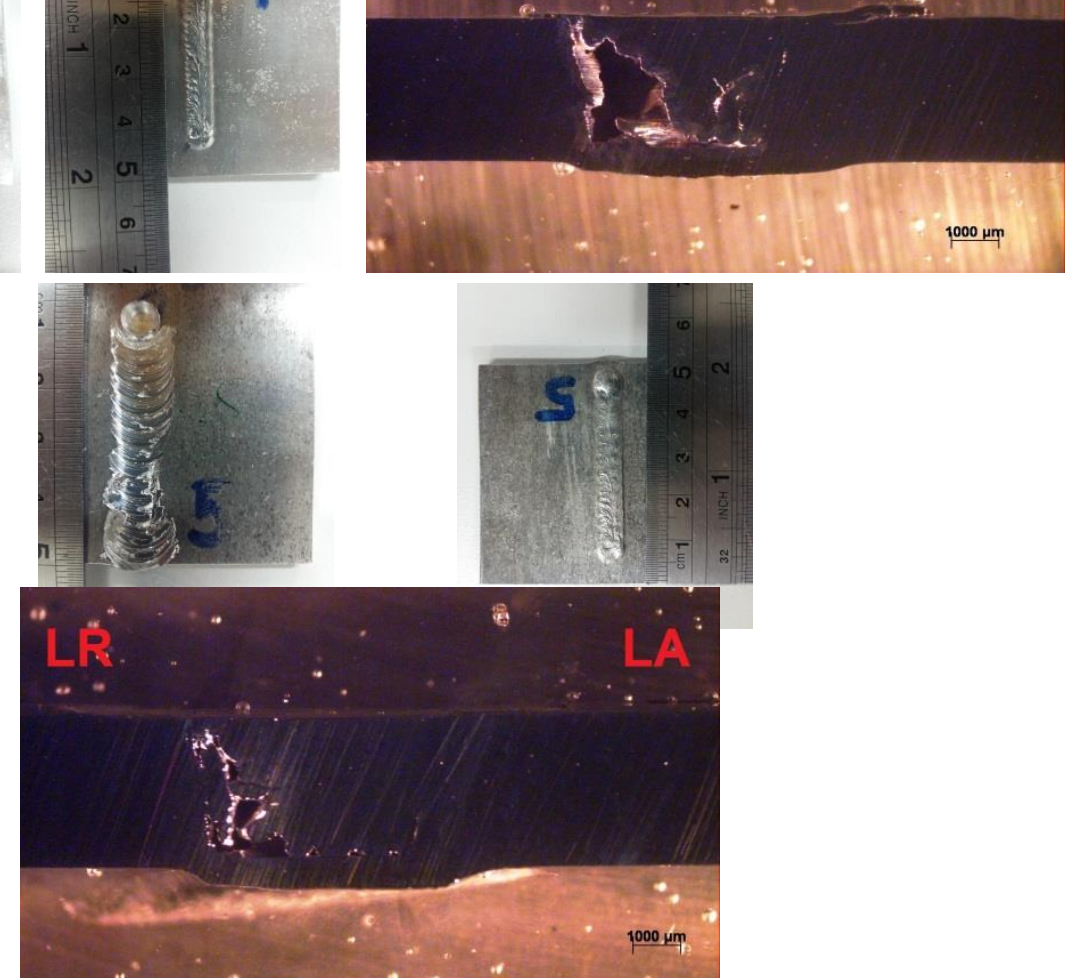

Figura 4. Aspecto superficial superior e inferior, e transversal das amostras do bloco 1: (a) Amostra A1/350/50/3/0, (b) Amostra A4/350/50/3/5 e (c) Amostra A5/350/50/3/10.

As macrografias apresentadas na figura 5 representam as inspeções visuais de superfície e raiz das amostras 4 e 2 (Bloco 2 - Tabela 3), assim como a macroestrutura com ampliação de 8x.

Para as amostras do bloco 2, foi possível verificar que aumentos na velocidade de avanço (VA) da ferramenta no material, provocou uma tendência positiva na mistura na zona do nugget, resultado este não esperado. Pois aumentos na velocidade de avanço da ferramenta costumam provocar uma tendência negativa no gradiente de temperatura local [2]. Para a análise da descontinuidade assimétrica, cabe a mesma observação destacada para o bloco1.

Tabela 3. Parâmetros bloco 2 com variação na velocidade de avanço da ferramenta

\begin{tabular}{lccccc}
\hline Nomenclatura & Amostra & RPM & $\begin{array}{c}\text { VA } \\
\text { (mm/min) }\end{array}$ & $\begin{array}{c}\text { Inclinação } \\
\text { (Graus) }\end{array}$ & $\begin{array}{c}\text { Profundidade de } \\
\text { Penetração da } \\
\text { ferramenta (mm) }\end{array}$ \\
\hline A4/350/50/3/5 & 4 & 350 & $\mathbf{5 0}$ & 3 & 0,05 \\
\hline A2/350/50/3/5 & 2 & 350 & $\mathbf{1 0 0}$ & 3 & 0,05 \\
\hline
\end{tabular}


(a)

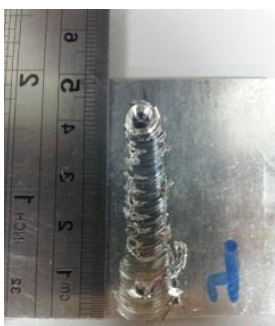

(b)

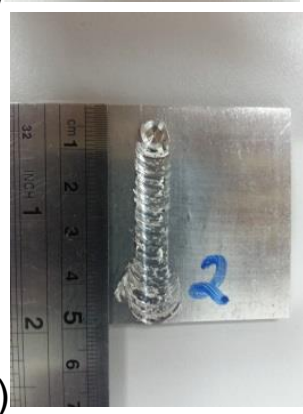

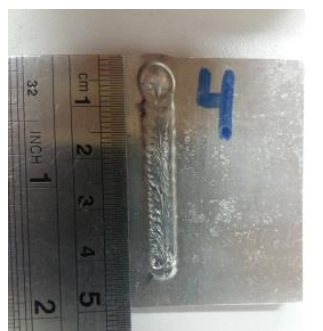
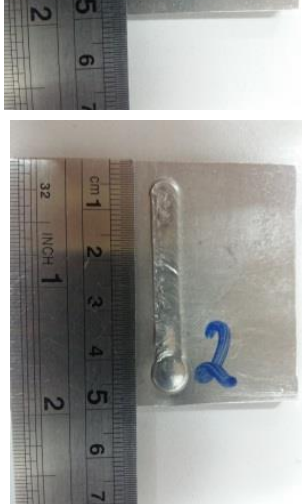
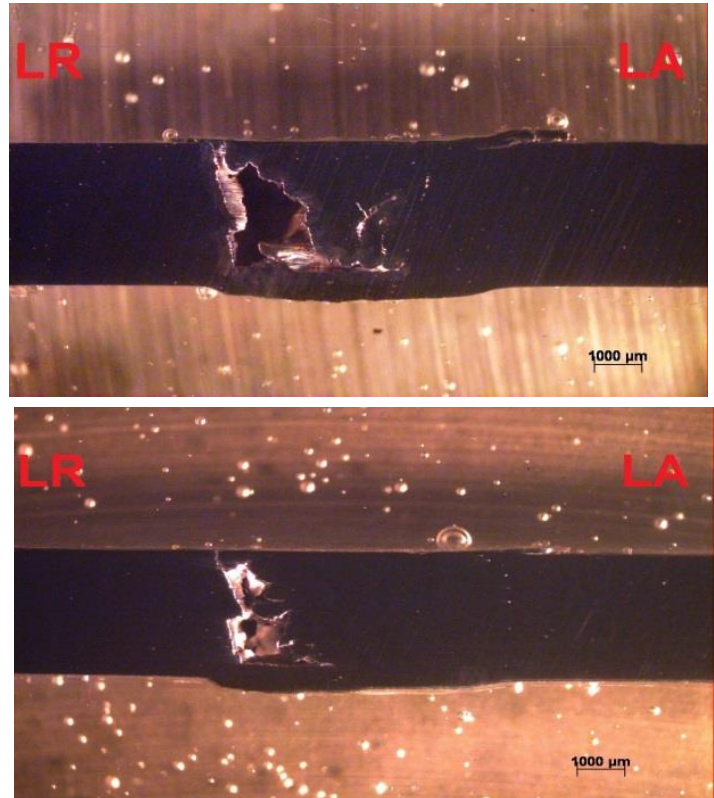

Figura 5. Aspecto superficial superior e inferior, e transversal das amostras do bloco 2: (a) Amostra A4/350/50/3/5 e (b) Amostra A2/350/50/3/5.

As macrografias apresentadas na figura 6 representam as inspeções visuais de superfície e raiz das amostras 3 e 2 (Bloco 3 - Tabela 4), assim como a macroestrutura com ampliação de $8 x$.

Tabela 4. Parâmetros bloco 3 com variação na rotação por minuto da ferramenta.

\begin{tabular}{lccccc}
\hline Nomenclatura & Amostra & RPM & $\begin{array}{c}\text { VA } \\
\text { (mm/min) }\end{array}$ & $\begin{array}{c}\text { Inclinação } \\
\text { (Graus) }\end{array}$ & $\begin{array}{c}\text { Profundidade de } \\
\text { Penetração da } \\
\text { ferramenta (mm) }\end{array}$ \\
\hline A3/500/50/3/5 & 3 & $\mathbf{5 0 0}$ & 100 & 3 & 0,05 \\
\hline A2/350/50/3/5 & 2 & $\mathbf{3 5 0}$ & 100 & 3 & 0,05 \\
\hline
\end{tabular}

(a)

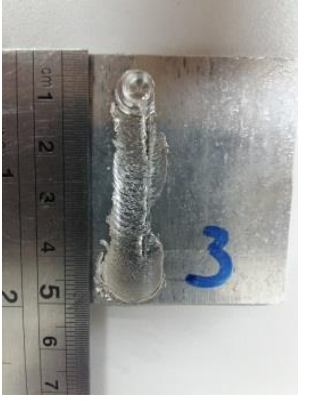

(b)

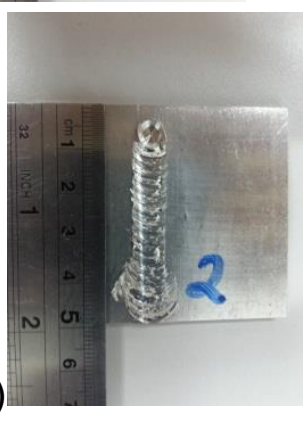

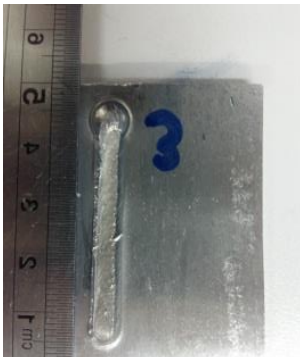

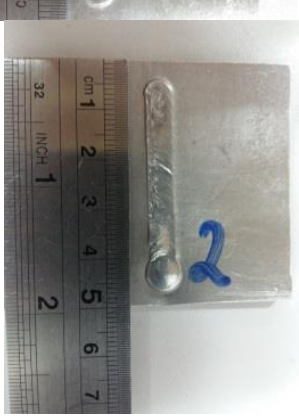

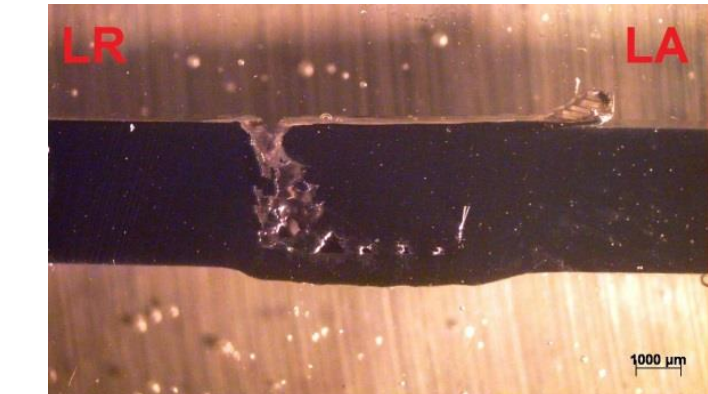

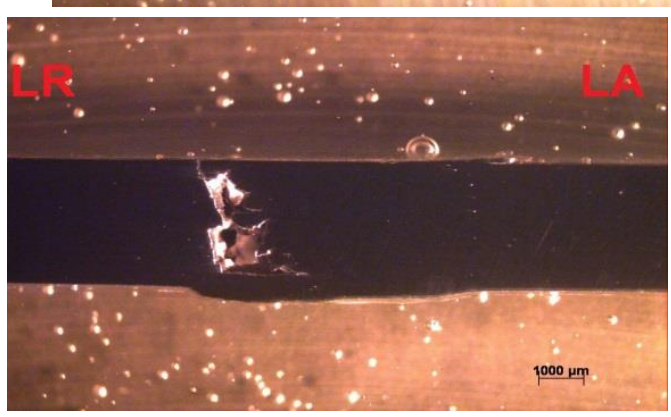

Figura 6. Aspecto superficial superior e inferior, e transversal das amostras do bloco 3: (a) Amostra Amostra A3/500/100/3/5 e (b) Amostra A2/350/100/3/5. 
Para as amostras do bloco 3, foi possível verificar que aumentos no RPM da ferramenta, provocaram uma tendência negativa na mistura na zona do nugget, resultado este não esperado. Pois aumentos na rotação da ferramenta tendem a provocar uma tendência positiva no gradiente de temperatura local contribuindo assim de maneira positiva para a mistura do material na zona do nugget [2]. Para a análise da descontinuidade assimétrica, cabe a mesma observação destacada para o bloco1.

As macrografias apresentadas na figura 7, representam as inspeções visuais de superfície e raiz das amostras 7 e 6 (Bloco 4 - Tabela 5), assim como a macroestrutura com ampliação de 8x.

Tabela 5. Parâmetros bloco 4 com variação na inclinação da ferramenta.

\begin{tabular}{lccccc}
\hline Nomenclatura & Amostra & RPM & $\begin{array}{c}\text { VA } \\
\text { (mm/min) }\end{array}$ & $\begin{array}{c}\text { Inclinação } \\
\text { (Graus) }\end{array}$ & $\begin{array}{c}\text { Profundidade de } \\
\text { Penetração da } \\
\text { ferramenta (mm) }\end{array}$ \\
\hline A7/500/50/0/0 & 7 & 500 & 50 & $\mathbf{0}$ & 0,00 \\
\hline A6/500/50/3/0 & 6 & 500 & 50 & $\mathbf{3}$ & 0,00 \\
\hline
\end{tabular}

(a)
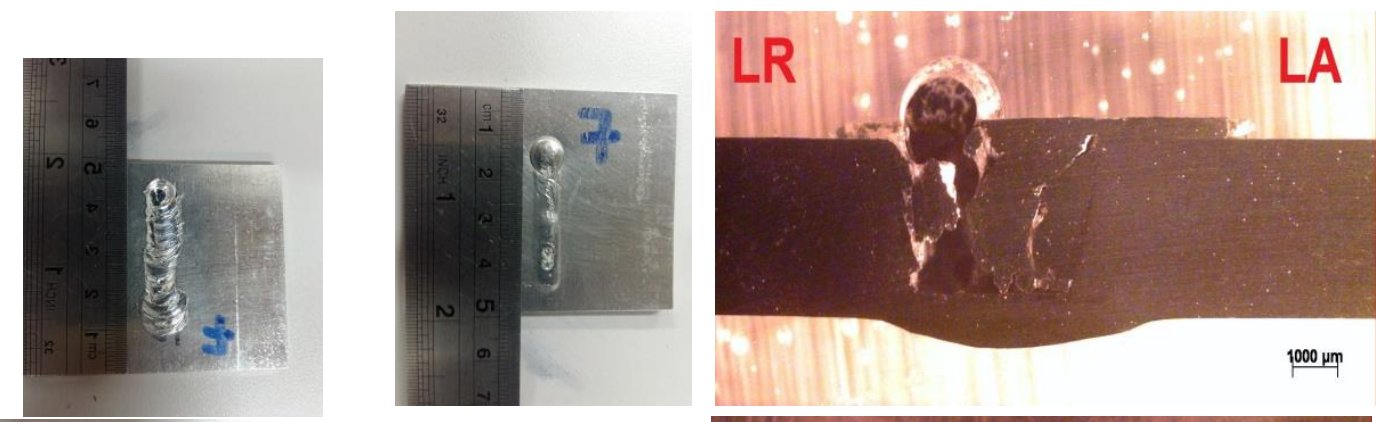

(b)
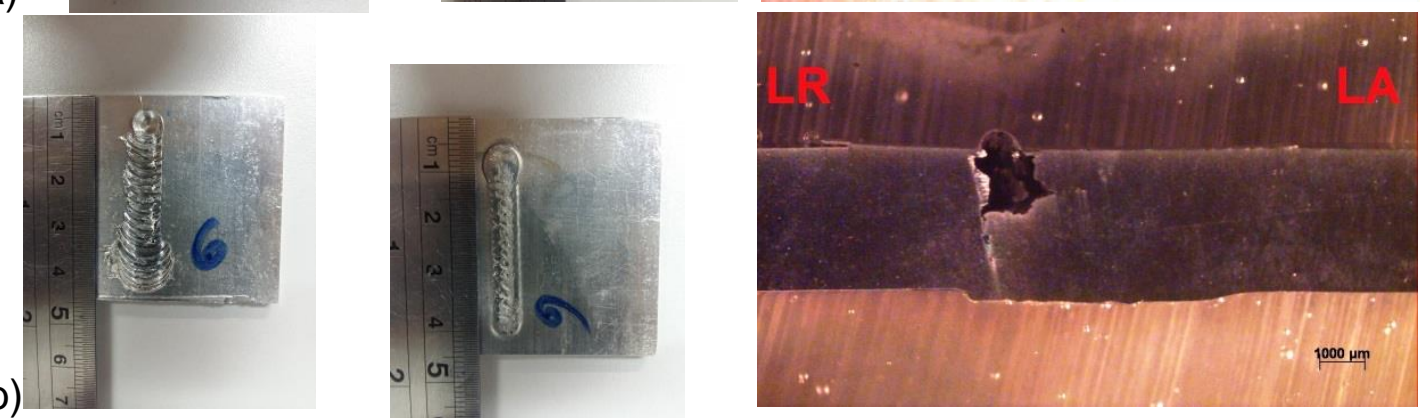

Figura 7. Aspecto superficial superior e inferior, e transversal das amostras do bloco 4: (a) Amostra Amostra A7/500/50/0/0 e (b) Amostra A6/500/50/3/0.

Para as amostras do bloco 4, foi possível verificar que a redução na inclinação da ferramenta provocou uma tendência negativa na mistura da zona do nugget, resultado este esperado. Pois reduções inferiores a $3^{\circ}$ na inclinação da ferramenta provocam uma tendência negativa [2], uma inclinação adequada do pino da ferramenta no sentido da direção de arrasto, garante com que o ombro da ferramenta mantenha o material agitado devido ao contado entre ombro e o material extrudado para cima da zona de mistura. Para a análise da descontinuidade assimétrica, cabe a mesma observação destacada para o bloco 1.

As macrografias apresentadas na figura 8 representam as inspeções visuais de superfície e raiz das amostras 6 e 8 (Bloco 5 - Tabela 6), assim como a macroestrutura com ampliação de 8x. 
Tabela 6. Parâmetros bloco 5 com variação na rotação da ferramenta.

\begin{tabular}{lccccc}
\hline Nomenclatura & Amostra & RPM & $\begin{array}{c}\text { VA } \\
\text { (mm/min) }\end{array}$ & $\begin{array}{c}\text { Inclinação } \\
\text { (Graus) }\end{array}$ & $\begin{array}{c}\text { Profundidade de } \\
\text { Penetração da } \\
\text { ferramenta (mm) }\end{array}$ \\
\hline A6/500/50/3/0 & 6 & $\mathbf{5 0 0}$ & 50 & 3 & 0,00 \\
\hline $\mathrm{A} 8 / 1000 / 50 / 3 / 0$ & 8 & $\mathbf{1 0 0 0}$ & 50 & 3 & 0,00 \\
\hline
\end{tabular}

(a)

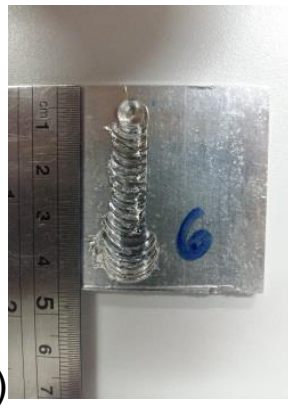

(b)

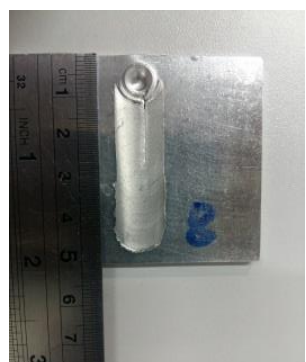

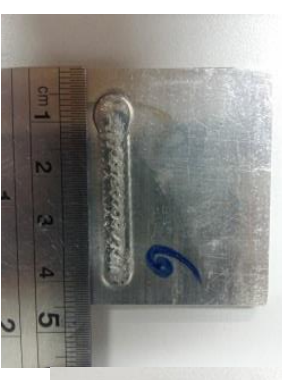

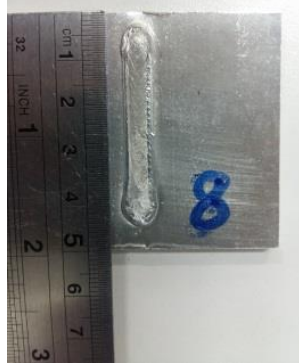

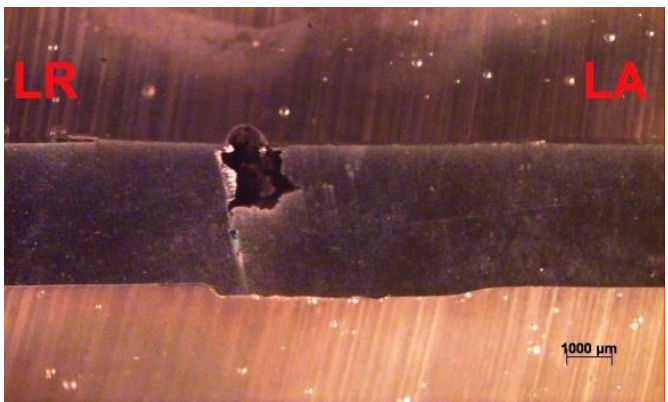

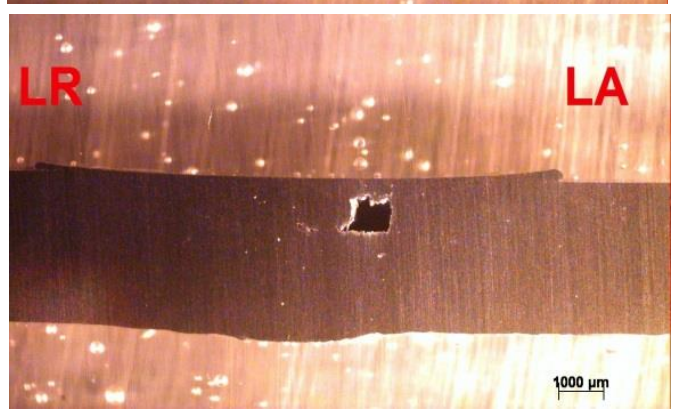

Figura 8. Aspecto superficial superior e inferior, e transversal das amostras do bloco 5: (a) Amostra Amostra A6/500/50/3/0 e (b) Amostra A8/1000/50/3/0.

Para as amostras do bloco 5 , foi possível verificar que o aumento na rotação da ferramenta provocou uma tendência positiva na mistura da zona do nugget, resultado este esperado. Pois aumentos na rotação da ferramenta provoca uma tendência positiva no gradiente de temperatura local contribuindo assim de maneira positiva para a mistura do material na zona do nugget [2]. Resultado este que diverge do bloco 3 . Para a análise da descontinuidade assimétrica, cabe a mesma observação destacada para o bloco1.

Todas as amostras de A1 a A8 apresentaram descontinuidades durante as inspeções visuais de superfície e de raiz, assim como a presença de macro defeitos internos. A partir da amostra $A 9 / 1000 / 50 / 3 / 5$, não foram mais encontrados nenhum tipo de descontinuidade na superfície e/ou na raiz do material inspecionado em função das variações de parâmetros. No entanto, a presença de macro defeitos internos ainda se faziam presentes no material quando seccionado transversalmente, conforme Figuras 10 e 11.

As macrografias apresentadas na figura 9 representam as inspeções visuais de superfície e raiz das amostras 8 e 9 (Bloco 6 - Tabela 7), assim como a macroestrutura com ampliação de $8 x$.

Tabela 7. Parâmetros bloco 6 com variação na profundidade de penetração da ferramenta.

\begin{tabular}{cccccc}
\hline Nomenclatura & Amostra RPM & $\begin{array}{c}\text { VA } \\
(\mathbf{m m} / \mathbf{m i n})\end{array}$ & $\begin{array}{c}\text { Inclinação } \\
\text { (Graus) }\end{array}$ & $\begin{array}{c}\text { Profundidade de } \\
\text { Penetração da } \\
\text { ferramenta (mm) }\end{array}$ \\
\hline $\mathrm{A} 8 / 1000 / 50 / 3 / 0$ & 8 & 1000 & 50 & 3 & $\mathbf{0 , 0 0}$ \\
\hline $\mathrm{A} 9 / 1000 / 50 / 3 / 5$ & 9 & 1000 & 50 & 3 & $\mathbf{0 , 0 5}$ \\
\hline
\end{tabular}


(a)
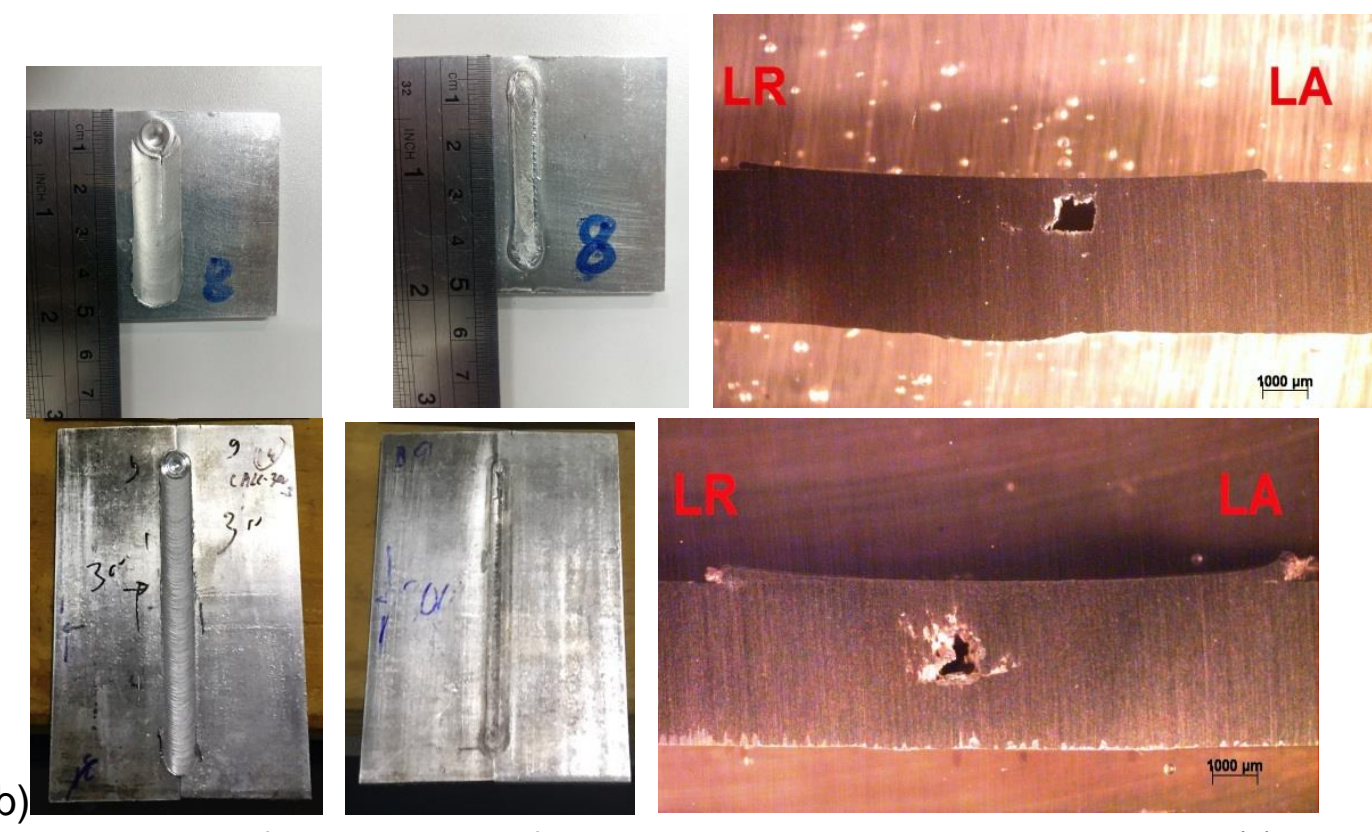

Figura 9. Aspecto superficial superior e inferior, e transversal das amostras do bloco 6: (a) Amostra Amostra A8/1000/50/3/0 e (b) Amostra A9/1000/50/3/5.

Para as amostras do bloco 6, foi possível verificar que aumentos na profundidade de penetração, da ferramenta no material, provocou uma tendência positiva na mistura da zona do nugget. Para a análise da variável profundidade de penetração cabe a mesma conclusão do bloco1. A partir dos valores utilizados nas variáveis da amostra A9, não foram mais encontradas descontinuidades na superfície do material. Para as amostras dos blocos 7, 8 e 9 (Tabela 8), buscou-se eliminar o defeito interno de volume na zona de mistura modificando os valores das variáveis: velocidade de avanço, RPM e profundidade de penetração da ferramenta.

Tabela 8. Parâmetros blocos 7, 8 e 9.

\begin{tabular}{|c|c|c|c|c|c|}
\hline Nomenclatura & Amostra & RPM & $\begin{array}{c}\text { VA } \\
(\mathrm{mm} / \mathrm{min})\end{array}$ & $\begin{array}{l}\text { Inclinação } \\
\text { (Graus) }\end{array}$ & $\begin{array}{l}\text { Profundidade de } \\
\text { Penetração da } \\
\text { ferramenta ( } \mathrm{mm})\end{array}$ \\
\hline \multicolumn{6}{|c|}{ Bloco 7} \\
\hline A9/1000/50/3/5 & 9 & 1000 & 50 & 3 & 0,05 \\
\hline $\mathrm{A} 10 / 1000 / 50 / 3 / 5$ & 10 & 1000 & 100 & 3 & 0,05 \\
\hline \multicolumn{6}{|c|}{ Bloco 8} \\
\hline $\mathrm{A} 10 / 1000 / 50 / 3 / 5$ & 10 & 1000 & 100 & 3 & 0,05 \\
\hline A11/1500/50/3/5 & 11 & 1500 & 100 & 3 & 0,05 \\
\hline \multicolumn{6}{|c|}{ Bloco 9} \\
\hline A11/1500/50/3/5 & 11 & 1500 & 100 & 3 & 0,05 \\
\hline A12/1500/50/3/5 & 12 & 1500 & 100 & 3 & 0,08 \\
\hline
\end{tabular}

Pode-se observar que as intervenções nas amostras A10, A11 e A12 (Figuras 10b, 11a e 11b), surtiram efeito positivo, porém não o suficiente para a eliminação do defeito de volume na zona de mistura.

Como um último bloco a ser apresentado, têm-se os resultados entre 04 amostras com RPM's distintos, conforme Tabela 9 e macrografias anteriormente apresentadas nas Figuras 6, 10b e 11a. 
(a)

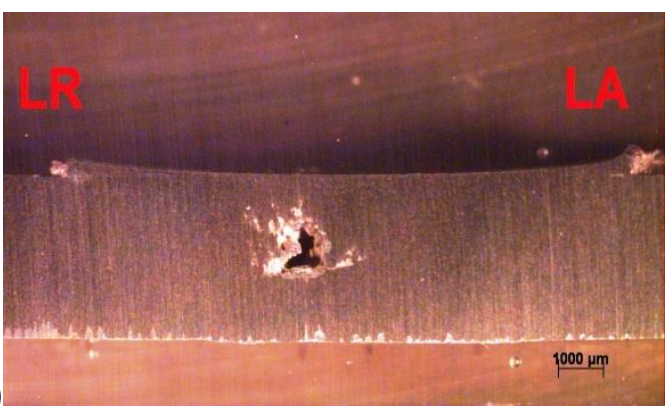

(b)

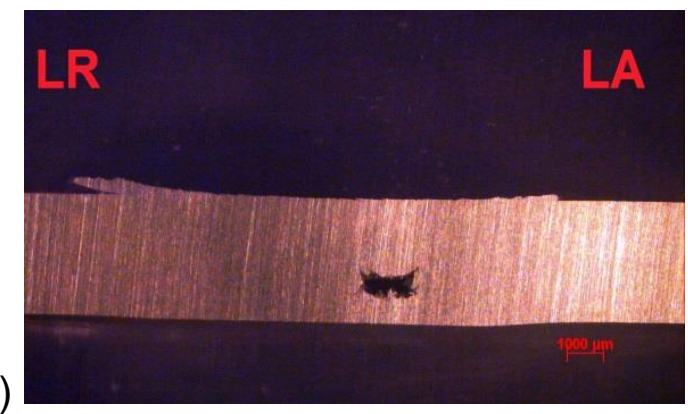

Figura 10. Aspecto transversal das amostras (a) Amostra A9/1000/50/3/5 - bloco 7 e (b) Amostra A10/1000/100/3/5 - blocos 7 e 8.

(a)

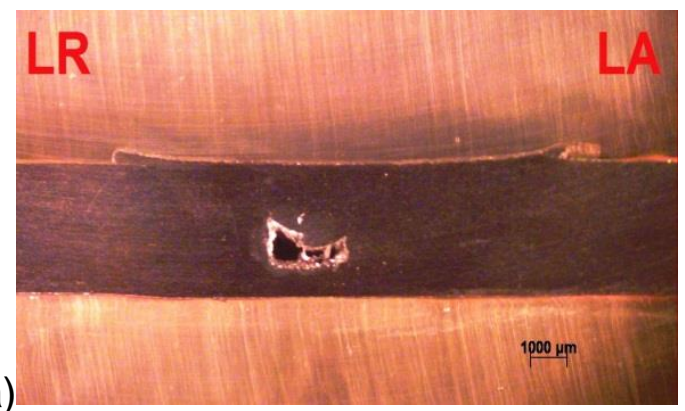

11. Aspecto transversal das amostras (a) amostra A12/1500/100/3/8 - bloco 9.

Tabela 9. Parâmetros bloco 10 com variação na rotação da ferramenta

\begin{tabular}{lccccc}
\hline Nomenclatura & Amostra & RPM & $\begin{array}{c}\text { VA } \\
\text { (mm/min) }\end{array}$ & $\begin{array}{c}\text { Inclinação } \\
\text { (Graus) }\end{array}$ & $\begin{array}{c}\text { Profundidade de } \\
\text { Penetração da } \\
\text { ferramenta (mm) }\end{array}$ \\
\hline $\mathrm{A} 2 / 350 / 50 / 3 / 5$ & 2 & $\mathbf{3 5 0}$ & 100 & 3 & 0,05 \\
\hline $\mathrm{A} 3 / 500 / 50 / 3 / 5$ & 3 & $\mathbf{5 0 0}$ & 100 & 3 & 0,05 \\
\hline $\mathrm{A} 10 / 1000 / 50 / 3 / 5$ & 10 & $\mathbf{1 0 0 0}$ & 100 & 3 & 0,05 \\
\hline $\mathrm{A} 11 / 1500 / 50 / 3 / 5$ & 11 & $\mathbf{1 5 0 0}$ & 100 & 3 & 0,05 \\
\hline
\end{tabular}

É importante salientar que existem diversos fatores que podem influenciar o fluxo de material durante o processo por fricção e mistura (FSP). Esses fatores incluem a geometria da ferramenta e parâmetros de processamento, com isso é muito provável que o fluxo de material dentro do nugget durante o processo por fricção e mistura, seja influenciado por vários parâmetros independentes [1]. Sendo assim, uma análise mais detalhada a partir de um quantitativo maior de amostras deve ser feita para a obtenção de um resultado com um índice de confiabilidade adequado ao propósito de obtenção de um conjunto de varáveis ótimas para o processo por friç̧ão e mistura.

\section{CONCLUSÃO}

Em termos da influência das variáveis adotadas no FSP na formação de macro defeitos, conclui-se que:

- Para rotações de 350 RPM e 500 RPM observou-se sistematicamente a presença de defeitos de volume na zona de mistura (cavidades) de maneira assimétrica no lado de retrocesso da ferramenta, mesmo com reduções da velocidade de avanço e aumentos da penetração da ferramenta no material. Por tanto, não houve eficiência no atingimento da temperatura adequada de coalescência do material e deformação 
plástica (extrusão e forjamento) suficiente para a mistura mecânica do material na zona do nugget.

- Para rotações de 1000 RPM e 1500 RPM, a presença de defeitos de volume na zona de mistura (cavidades) apresentou significativa redução de seu tamanho, resultado este, gerado a partir do aumento da temperatura local devido ao aumento da rotação, assim como uma maior pressão do ombro da ferramenta na superfície do material, em virtude do aumento final para $0,08 \mathrm{~mm}$ de penetração do pino da ferramenta no material, causando assim uma maior efetividade na mistura, assim como um deslocamento do defeito de volume na zona de mistura (cavidades) para o centro do nugget, resultado este esperado devido à direção do fluxo de material na zona de mistura.

\section{Agradecimentos}

Os autores agradecem ao IME e ao IPT pela infraestrutura oferecida e equipamentos disponibilizados à pesquisa em questão. A CAPES pelo auxílio financeiro via bolsa de estudos de mestrando (da Silva, A. S.; Diniz, S. B.; Casanova, A. M. B.) e de doutorando (Diniz, S. B.; Casanova, A. M. B.).

\section{REFERÊNCIAS}

1 Li, Y.; Murr, L.E.; McClure, J.C.; Flow Visualization and Residual Microstructures Associated with The Friction Stir Welding of 2024 Aluminum to 6061 Aluminum; Materials Science and Engineering A; 1999; (271): 213-223.

2 Mishra, R.S.; Ma, Z.Y.; Friction Stir Welding and Processing; Materials Science and Engineering $R ; 2005 ;(50):$ 1-78.

3 Mishra, R.S.; Mahoney, M.W.; McFadden, S.X.; Mara, N.A.; Mukherjee, A.K.; High Strain Rate Superplasticity in a Friction Stir Processed 7075 Al Alloy; Scripta Materialia; 1999; (42): 163-168.

4 Mishra, R.S.; Mahoney, M.W.; Friction stir processing: A New Grain Refinement Technique to Achieve High Strain Rate Superplasticity in Commercial Alloys; Materials Science Forum; 2001; (507): 357-359.

5 Ma, Z.Y.; Mishra, R.S.; Mahoney, M.W.; Superplastic Deformation Behaviour of Friction Stir Processed 7075Al Alloy; Acta Materialia; 2002; (50): 4419-4430.

6 Berbon, P.B.; Bingel, W.H.; Mishra, R.S.; Bampton, C.C.; Mahoney,M.W.; Friction stir processing: a tool to homogenize nanocomposite aluminum alloys; Scripta Materialia; 2001; (44): 61-66.

7 Spowart, J.E; Ma, Z.Y.; Mishra, R.S.; Jata, K.V.; Mahoney, M.W.; Semiatin, S.L.; Friction Stir Welding and Processing II; TMS; 2003; 243-252.

8 Ma, Z.Y.; Sharma, S.R.; Mishra, R.S.; Manohey, M.W.; Microstructural modification of cast Aluminum alloys via friction stir processing; Materials Science Forum; 2003; (426432): 2891-2896.

9 James, M. N.; Bradley, G. R.; Lombard, H.; Hattingh, D. G.; The Relationship Between Process Mechanisms and Crack Paths in Friction Stir Welded 5083-H321 and 5383H321 Aluminium Alloys; Fatigue and Fracture of Engineering Materials and Structures; 2005; (28): 245-256.

10 Lomolino, S.; Tovo, R.; Santos, J.; On The Fatigue Behaviour and Design Curves of Friction Stir Butt-Welded Al alloys; International Journal of Fatigue; 2005; (27): 305-316.

11 Jin, Z.; Beaudoin, A.; Bieler, T.A.; Radhakrishnan, B.; Hot Deformation of Aluminum Alloys III; TMS; 2003; 313-352.

12 Genicolo, M. A. C.; Estudo de Viabilidade para a Implementação de Uniões Soldadas pelo Método Friction Stir Welding em Estruturas Aeronauticas; USP; 2007; 26-27. 